\section{[gw22-e0157] DISTRIBUTION CHARACTERISTICS OF DOMINANT FREQUENCIES IN VENTRICULAR FIBRILLATION WITH DIFFERENT DEFIBRILLATION THRESHOLDS: A RETROSPECTIVE ANALYSIS OF ELECTROCARDIOGRAM}

Li Jingsha, Zhong Jingquan, Zeng Qixian, Liu Hongzhen, Meng Xianglin, Liu Donglin, Su Guoying, Zhang Yun Qilu Hospital, Shandong University, Jinan, China

10.1136/heartjnl-2011-300867.4

Aim Ventricular fibrillation (VF) is normally a sustained condition, and electrical shock is required to end it. In the laboratory, we found that the defibrillation thresholds of VF lasting for the same time may be different even in similar healthy animals. The specific electrophysiological mechanisms determine the defibrillation thresholds are unknown but profoundly interesting, since they may provide information regarding VF mechanisms and aid in the development of new algorithms in implantable cardioverter defibrillators (ICDs) to avoid treating fibrillations at improper energies. Retrospective out-ofhospital human VF studies have suggested that VF waveform characteristics such as mean frequency and amplitude could predict the duration of VF accurately and the possibility of successful defibrillation. However, dominant frequency (DF) extracted by fast Fourier transform (FFT) remains the most widely reported feature of VF. The study aimed to quantify the distribution of DFs of the VF waveform in VF episodes with different defibrillation thresholds.

Methods VF episodes successfully defibrillated respectively at $70 \mathrm{~J}(\mathrm{n}=4), 100 \mathrm{~J}(\mathrm{n}=4)$, the first $150 \mathrm{~J}(\mathrm{n}=5)$ and not successfully defibrillated $(n=4)$ were selected from our previous swine model of 8 min VF. DFs and their corresponding signal amplitudes in each $10 \mathrm{~s}$ interval of electrogram were obtained using spectral analysis transformed by FFT. Dominant frequency ratio (DFR) was defined as the ratio of the maximum and minimum DF and analysed in each 1 min segment.

Results The DFRs were smaller in VF with lower defibrillation thresholds and they were the largest in the defibrillation-failure VF (70 J: $1.16 \pm 0.11$ vs $100 \mathrm{~J}: 1.20 \pm 0.14$ vs $150 \mathrm{~J}$ : $1.26 \pm 0.12$ vs Failure: $1.46 \pm 0.24, p=0.005)$. Both DFs and the signal amplitudes were higher in VF with lower defibrillation thresholds (70 J: $11.76 \pm 1.27 \mathrm{~Hz}$ vs $100 \mathrm{~J}: 10.34 \pm 1.41 \mathrm{~Hz}$ vs 150 J: $9.99 \pm 1.75 \mathrm{~Hz}$ vs Failure: $8.79 \pm 4.03 \mathrm{~Hz}$, respectively, $\mathrm{p}=0.000$ and $70 \mathrm{~J}: 2.79 \pm 1.15 \mathrm{mV} \mathrm{Hz}$ vs $100 \mathrm{~J}: 2.26 \pm 0.57 \mathrm{mV} \mathrm{Hz}$ vs 150 J: $1.74 \pm 0.66 \mathrm{mV} \mathrm{Hz}$ vs Failure: $0.88 \pm 0.23 \mathrm{mV} \mathrm{Hz}$, respectively, $\mathrm{p}=0.000)$.

Conclusion The present study showed that the spectral characteristics of the VF waveform from surface ECG were different in VF with different defibrillation thresholds. VF with smaller volatility of DF values, higher DFs and higher signal amplitudes had a lower defibrillation threshold. Therefore, VF waveform characteristics were not merely a simple reflection of VF duration and a valid tool for predicting the appropriateness of resuscitation efforts with chest compressions first or shock first but also could be used to predict the proper defibrillation energy especially the first defibrillation energy. Thus, inadequate or excessive delivery of energy may be avoided. 